\title{
A Statistical Study of Trends of Rice-Wheat Production on Districts of Western U.P., India
}

\author{
Gopal Krishna Tiwari", Sri Sunil Kumar, Anvesha Singh and Babulal \\ Department of Agricultural Statistics, Narendra Deva University of Agriculture and \\ Technology, Kumarganj-Ayodhya (UP), 224-229, India \\ *Corresponding author:
}

Keywords

Rice, Wheat, Growth, Trend, Tabular Representation, Simple Growth Rate (SGR), Compound Growth Rate (CGR)

Article Info

Accepted:

18 May 2019

Available Online:

10 June 2019

\section{A B S T R A C T}

Rice (Oryza sativa L.) and Wheat (Triticum aestivum L.) are important constituents of Indian agriculture and nutritional security due to their high yield, economic viability, ability to generate on-farm and off-farm employment so this makes it one of the most important cereal crops in India main source of vitamins and minerals such as thiamine, niacin, iron, riboflavin, vitamin $\mathrm{D}$, calcium, and fiber. Wheat covers approximately $25 \%$ of the total global area devoted to by cereal crops India is the world's $2^{\text {nd }}$ largest producer with approximately 43 mha planted area, accounting for $22 \%$ of the world's rice production. Since the consumption of rice is directly proportional to the population growth. Feeding burgeoning population through the next 20-30 years remains a challenging task. Increasing domestic demand owing to population growth should meet the future challenges of food and nutritional security. On this prospective for the planning purposes decision to invest accordingly for the short and long-term plans, the present study has been undertaken to evaluate the growth in production, area and productivity of Rice and Wheat crops in Top 5 Rice-Wheat Producing districts of Western Uttar Pradesh, India by using tabular analysis and different growth models.

\section{Introduction}

Agriculture in India has a significant history. Today India ranks second worldwide in farm output. India is the second largest producer of wheat and rice, the world's major staple food. The government revised upwards total food grain production estimates by over one million tonne to a record 264.38MT for the agricultural year 2013-14 crop years on account of record wheat and rice production. Uttar Pradesh state is situated in North India.
Uttar Pradesh is fourth largest state in the country. State covers an area of about 243290 square $\mathrm{km}$ and possesses varied topographic features ranging from plain to vindhyan hills. Uttar Pradesh is divided in two physiographic regions viz. Gangatic plain and Southern Plateau. Agriculture is the most important occupation in the state as about $80 \%$ of its population resides in rural areas and $75 \%$ of the total workers are involved directly or indirectly in Farming which accounts for $27 \%$ of state's GDP. The state is divided into 4 
regions, namely Western (26 districts), Eastern (28 districts) Central (13 districts) and Bundelkhand (08 districts). At present state have 75 districts, 327 tehsils, 822 blocks and 107452 revenue villages. The important crops include Rice, wheat, maize, sugarcane, potato, etc. Majority of the agricultural land is used to grow major cereal crops: rice $\&$ wheat.

\section{Rice}

Rice production in India is an important part of the national economy. India is an important part of the national economy. India is the world's $2^{\text {nd }}$ largest producer with approximately 43 mha planted area, accounting for $22 \%$ of the world's rice production.

Rice (Oryza sativa), one of the three most Important food grain crops in world, forms the staple diet of 2.7 billion people. Its cultivation is of immense importance to food security of Asia, where more than $90 \%$ of the global rice is produced and consumed. The productivity and area of rice has declined during 2000-01 to 2010-11 in all regions of state.

Rice belongs to the genus Oryza, sub tribe oryzineae of the family Gramineae. There are 21 recognized species in genus Oryza.

Rice is grown in Uttar Pradesh during Kharif season from June to October/November. Rice is grown in all types of soils and are most suited for its cultivation. The traditional rice farming systems in India broadly include wetland \{lowland and upland\} system.

The life cycle can be divided into 3 important phases, i.e. vegetative, reproductive and ripening. Rice produces $32-59$ per cent of the dietary energy and 25-40 per cent of dietary protein in 39 countries. Annually rice is grown in 44.6 million hectares under 4 major ecosystems, irrigated (21 million ha), rainfed low land (14 million ha), rainfed upland (6 million ha) and flood prone (3 million ha). More than half of rice area (55per cent) is rain fed and distribution wise 80 per cent of the rain fed rice area is in eastern India making its vulnerable to vagaries of monsoon.

Uttar Pradesh is one of the major states of rice growing in the country. It accounts about 13.53 and 13.54 per cent towards the total acreage and production of the rice in the country (2011-12).

Rice production in India - 112.91 MT

Rice production in UP - 140.22 lakh tons

\section{Wheat}

Wheat is the world's most widely cultivated food crop. In India it is the second important staple food crop. It is over an area of about 215 million hectares with a production of 584 million tons of grain (1999). It is called as "King of cereals".

Per cent of gross cropped area in 2013-2014 is 40.55 .

Wheat crop needs clay loam or loam texture and moderate water holding capacity soil and these features are found in Eastern Uttar Pradesh so this region is suitable for wheat production.

When cultivation of high yielding dwarf varieties of wheat is done with improved scientific methods, they produce about 45-55 quintals of grain per hectare under irrigated conditions and 20-25 quintals per hectare under rainfed conditions. Wheat contains gluten which is essential for baking. For safe storage moisture content of grain should be 10-12 percent.

Wheat production in India- 99.70 million tons 


\section{Materials and Methods}

The present study was primarily based on the time series secondary data Production, Area and Productivity of Rice -Wheat crops in districts of western Uttar Pradesh in hectare, tonne, $\mathrm{kg} / \mathrm{ha}$ from 1996-97 to 2014- 15 are extracted from the published sources like Sankhikiya Patrika, Agricultural Statistics at a Glance, etc.

Effect of change in area and productivity on differential production of crops

An attempt has been made to study the effect of change in area and productivity of crops on differential production between two points of time.

Let $\mathrm{P}, \mathrm{A}$ and $\mathrm{Y}$ be the production, area and productivity of a particular crop at a given point of time. The $\mathrm{P}$ can be expressed as

$\mathrm{P}=\mathrm{Y} . \mathrm{A}$

Let $\Delta \mathrm{P}, \Delta \mathrm{A}$ and $\Delta \mathrm{Y}$ be the change in production, area and productivity of the crop after specific period of time. So we have

$\mathrm{P}+\Delta \mathrm{P}=(\mathrm{A}+\Delta \mathrm{A})(\mathrm{Y}+\Delta \mathrm{Y})$

$\mathrm{P}+\Delta \mathrm{P}=\mathrm{AY}+\Delta \mathrm{AY}+\mathrm{A} \Delta \mathrm{Y}+\Delta \mathrm{A} \Delta \mathrm{Y}$

Therefore we have

$\Delta \mathrm{P}=\Delta \mathrm{AY}+\mathrm{A} \Delta \mathrm{Y}+\Delta \mathrm{A} \Delta \mathrm{Y}$

Thus the total differential production consists of three components:

$\Delta \mathrm{AY}=$ Effect of change in area of crop

$\mathrm{A} \Delta \mathrm{Y}=$ Effect of change in productivity of crop

$\Delta \mathrm{A} \Delta \mathrm{Y}=$ Interaction effect due to change in area and productivity of crop.

\section{Trend and growth rate}

The trend and growth rate in area, production and productivity of different food grain crops have been worked out by fitting the following five different functions:

Simple linear function

$Y_{t}=a+b_{t}+\mu_{t}$

Compound function

$Y_{t}=a_{t}(1+r)^{t} \mu_{t}$

Where,

$\mathrm{Y}_{\mathrm{t}}$ : Time series data on area/production/productivity of different food grain crops at time $t, a \& b$ are parameters of the function to be estimated.

$\mathrm{t}$ : Time index $(\mathrm{t}=1,2, \ldots . \mathrm{n})$

r: Average compound growth rate per annum.

$\mu_{t}$ : error term at $t$ and is assumed to follow independently distributed

However, before the fitting above functions, the time series data on area and production were smoothed by three years movingaverage method.

\section{Computation of growth rate}

\section{For linear function}

After fitting the linear trend function by leastsquare method, we get the estimate of $b$ denoted by $\hat{b}$ (say). Then, annual linear simple growth rate (S.G.R.) is computed as follows

$\operatorname{SGR}(\%)=\frac{\hat{b}}{\bar{Y}} \times 100$ 
Where, $\bar{Y}$ is arithmetic mean of $Y_{t}$.

\section{Compound growth rate}

To obtain annual compound growth rate, the third function was first linearised by taking natural $\log$ on both side, i.e.

$\log Y_{t}=\log a+t \log (1+r)$ or $Y_{t}^{*}=a^{*}+b t$

Where, $\mathrm{Y}_{\mathrm{t}}^{*}=\log \mathrm{Y}_{\mathrm{t}}, \mathrm{a}^{*}=\log \mathrm{a}$ and $\mathrm{b}=\log$ $(1+r)$

The above linearized function was fitted by least square method and estimate of $b$ as $\hat{b}$ was obtained.

The annual compound growth rate is then computed as

$\operatorname{CGR}(\%)=($ antilog of $\hat{b}-1) \times 100$

All growth rates are expressed in percentage $(\%)$. The best fitted function was judged on the basis of $\mathrm{R}^{2}$ (coefficient of determination).

\section{Results and Discussion}

The agriculture sector is targeted to grow at the rate of 3-4\%. The results are presented and discussed objective- wise

\section{The First Objective}

To find out general profile of districts of western UP by way of tabular and graphical representation

An attempt has been made in this section to capture general features of districts of Western U.P.
Perusal of above Table 1(a) shows that: Highest percent change shown by Agra district in Rice followed by Bulandshr and Aligarh district.

Highest percent change shown by Mainpuri district in wheat followed by Pilibhit and Bijnor district.

Production of Rice declined being maximum in Muzzafarnagar followed by Amroha and Ghaziabad.

Production of wheat declined being maximum in Ghaziabad followed by Moradabad and Muzaffarnagar.

The decline is caused by division of districts into new districts:

Hapur from Ghaziabad; Shamli from Muzaffarnagar and Sambhal from Moradabad

Perusal of above Table 1(b) shows that:

Highest percent change shown by Agra district in Rice followed by Bulandshr and Aligarh district.

Highest percent change shown by Rampur district in wheat followed by Mainpuri and Mathura district.

Area of Rice declined being maximum in Muzzafarnagar followed by Etah and Ghaziabad.

Area of Wheat declined being maximum in Ghaziabad followed by Muzaffarnagar and Etah.

The decline is caused by division of districts into new districts: Hapur from Ghaziabad; Shamli from Muzaffarnagar and Sambhal from Moradabad.

Perusal of above Table 1(c) shows that: 
Table.1 (a) Production (Metric Tonnes) of Rice and Wheat in districts of western UP based on 3 -years moving average

\begin{tabular}{|c|c|c|c|c|}
\hline & \multicolumn{2}{|c|}{$1997-98$} & \multicolumn{2}{|c|}{ 2013-14 } \\
\hline & Rice & Wheat & Rice & Wheat \\
\hline Saharanpur & 188423.33 & 358056.3 & $\begin{array}{l}151937.33 \\
(-19.36 \%)\end{array}$ & $\begin{array}{l}344857.7 \\
(-3.69 \%)\end{array}$ \\
\hline Baghpat & 9496.67 & 186202.7 & $\begin{array}{l}14202.00 \\
(49.55 \%)\end{array}$ & $\begin{array}{l}221480.7 \\
(18.95 \%)\end{array}$ \\
\hline Ghaziabad & 33097.67 & 270786.3 & $\begin{array}{l}24538.67 \\
(-25.86 \%)\end{array}$ & $\begin{array}{c}106868.7 \\
(-60.53 \%)\end{array}$ \\
\hline Bulandshr & 39601.67 & 594606.3 & $\begin{array}{l}252242.00 \\
(536.00 \%)\end{array}$ & $\begin{array}{l}713631.3 \\
(20.02 \%)\end{array}$ \\
\hline Meerut & 41855.33 & 359986 & $\begin{array}{c}44690.33 \\
(6.77 \%)\end{array}$ & $\begin{array}{l}297099.3 \\
(-17.47 \%)\end{array}$ \\
\hline Muzaffarnagar & 104405.33 & 422996.7 & $\begin{array}{c}29283.67 \\
(-71.95 \%)\end{array}$ & $\begin{array}{c}267747.7 \\
(-36.70 \%)\end{array}$ \\
\hline Bareilly & 359743 & 467061.7 & $\begin{array}{c}353073.667 \\
(-1.85 \%)\end{array}$ & $\begin{array}{l}535351.7 \\
(14.62 \%)\end{array}$ \\
\hline Rampur & 303512 & 369385.3 & $\begin{array}{c}307092.33 \\
(1.18 \%)\end{array}$ & $\begin{array}{l}409203.3 \\
(10.78 \%)\end{array}$ \\
\hline Agra & 2239.67 & 413151.7 & $\begin{array}{c}19625.33 \\
(776.00 \%)\end{array}$ & $\begin{array}{c}437800.3 \\
(5.97 \%)\end{array}$ \\
\hline Mathura & 56165.67 & 529293 & $\begin{array}{l}115001.00 \\
(104.75 \%)\end{array}$ & $\begin{array}{l}605216.3 \\
(14.34 \%)\end{array}$ \\
\hline G.B. Nagar & 43332.67 & 197495.7 & $\begin{array}{c}55613 \\
(28.34 \%)\end{array}$ & $\begin{array}{c}143887 \\
(-27.14 \%)\end{array}$ \\
\hline Moradabad & 257832.00 & 524904 & $\begin{array}{l}220543.33 \\
(-14.46 \%)\end{array}$ & $\begin{array}{c}314175 \\
(-40.15 \%)\end{array}$ \\
\hline Amroha & 73433 & 251846.7 & $\begin{array}{c}51269 \\
(-30.18 \%)\end{array}$ & $\begin{array}{c}264627.3 \\
(5.07 \%)\end{array}$ \\
\hline Aligarh & 50116.33 & 615917 & $\begin{array}{l}203575.33 \\
(306.21 \%)\end{array}$ & $\begin{array}{l}689395.3 \\
(11.93 \%)\end{array}$ \\
\hline Firozabad & 28797.33 & 269601.3 & $\begin{array}{c}62628.33 \\
(117.48 \%)\end{array}$ & $\begin{array}{l}306217.7 \\
(13.58 \%)\end{array}$ \\
\hline Mainpuri & 127225.33 & 365411.7 & $\begin{array}{c}193468.33 \\
(52.07 \%)\end{array}$ & $\begin{array}{l}485240.7 \\
(32.79 \%)\end{array}$ \\
\hline Bijnor & 180181.67 & 303361.7 & $\begin{array}{l}138393.67 \\
(-23.19 \%)\end{array}$ & $\begin{array}{l}382622.7 \\
(26.13 \%)\end{array}$ \\
\hline Etah & 55827.33 & 437509.7 & $\begin{array}{c}52591 \\
(-5.80 \%)\end{array}$ & $\begin{array}{l}398997.7 \\
(-8.80 \%)\end{array}$ \\
\hline Hathras & 15913.67 & 252628 & $\begin{array}{c}54037.33 \\
(239.57 \%)\end{array}$ & $\begin{array}{c}264020.3 \\
(4.51 \%)\end{array}$ \\
\hline Shahjehanpur & 406395.00 & 699639.33 & $\begin{array}{c}561956.67 \\
(38.37 \%)\end{array}$ & $\begin{array}{l}766334 \\
(9.53 \%)\end{array}$ \\
\hline Pilibhit & 395400.00 & 382621.3 & $\begin{array}{c}418305.00 \\
(5.79 \%)\end{array}$ & $\begin{array}{l}484313.3 \\
(26.58 \%)\end{array}$ \\
\hline Badaun & 136500.00 & 742921.7 & $\begin{array}{c}180335.00 \\
(32.11 \%)\end{array}$ & $\begin{array}{c}730933 \\
(-1.61 \%)\end{array}$ \\
\hline
\end{tabular}


Table.1 (b) Area (Hectare) of rice and wheat in districts of western UP based on 3-years moving average

\begin{tabular}{|c|c|c|c|c|}
\hline & \multicolumn{2}{|c|}{$1997-98$} & \multicolumn{2}{|c|}{ 2013-14 } \\
\hline & Rice & Wheat & Rice & Wheat \\
\hline Saharanpur & 73163 & 125722.3 & $\begin{array}{l}60472.67 \\
(-17.35 \%)\end{array}$ & $\begin{array}{l}119511.3 \\
(-4.94 \%)\end{array}$ \\
\hline Baghpat & 3382.667 & 53071 & $\begin{array}{c}5152 \\
(52.31 \%)\end{array}$ & $\begin{array}{c}54423.33 \\
(2.55 \%)\end{array}$ \\
\hline Ghaziabad & 14272 & 70594.33 & $\begin{array}{c}9472.667 \\
(-33.63 \%)\end{array}$ & $\begin{array}{c}27589 \\
(-60.92 \%)\end{array}$ \\
\hline Bulandshr & 16256 & 170311.7 & $\begin{array}{c}97354 \\
(498.88 \%)\end{array}$ & $\begin{array}{l}197130.3 \\
(15.75 \%)\end{array}$ \\
\hline Meerut & 17087.67 & 83683 & $\begin{array}{c}15757 \\
(-7.79 \%)\end{array}$ & $\begin{array}{l}76836.67 \\
(-8.18 \%)\end{array}$ \\
\hline Muzaffarnagar & 39173.33 & 132976.3 & $\begin{array}{c}11900.33 \\
(-69.62 \%)\end{array}$ & $\begin{array}{c}80401.33 \\
(-39.54 \%)\end{array}$ \\
\hline Bareilly & 158659.7 & 180617.3 & $\begin{array}{l}162694 \\
(2.54 \%)\end{array}$ & $\begin{array}{c}202621 \\
(12.18 \%)\end{array}$ \\
\hline Rampur & 128491.3 & 114494.7 & $\begin{array}{c}135667.7 \\
(5.59 \%)\end{array}$ & $\begin{array}{c}142738 \\
(24.67 \%)\end{array}$ \\
\hline Agra & 1049.667 & 120948.3 & $\begin{array}{c}7789 \\
(642.05 \%)\end{array}$ & $\begin{array}{c}133714 \\
(10.55 \%)\end{array}$ \\
\hline Mathura & 26467.67 & 166463.3 & $\begin{array}{l}48053.67 \\
(81.56 \%)\end{array}$ & $\begin{array}{l}194610.3 \\
(16.91 \%)\end{array}$ \\
\hline G.B. Nagar & 15252 & 70223.67 & $\begin{array}{c}21518 \\
(41.08 \%)\end{array}$ & $\begin{array}{l}43032.33 \\
(-38.72 \%)\end{array}$ \\
\hline Moradabad & 107671.7 & 168822.3 & $\begin{array}{l}95825.67 \\
(-11.00 \%)\end{array}$ & $\begin{array}{c}121967.7 \\
(-27.75 \%)\end{array}$ \\
\hline Amroha & 29713 & 96307.67 & $\begin{array}{l}24791.67 \\
(-16.56 \%)\end{array}$ & $\begin{array}{l}92377.33 \\
(-4.08 \%)\end{array}$ \\
\hline Aligarh & 17581.67 & 190970 & $\begin{array}{c}90726.33 \\
(416.03 \%)\end{array}$ & $\begin{array}{l}223079.3 \\
(16.81 \%)\end{array}$ \\
\hline Firozabad & 21200 & 93157 & $\begin{array}{c}23416 \\
(10.45 \%)\end{array}$ & $\begin{array}{c}100073 \\
(7.42 \%)\end{array}$ \\
\hline Mainpuri & 60549.67 & 129929 & $\begin{array}{l}73778.33 \\
(21.85 \%)\end{array}$ & $\begin{array}{l}154020.7 \\
(18.54 \%)\end{array}$ \\
\hline Bijnor & 61495.33 & 113176.3 & $\begin{array}{c}55117 \\
(-10.37 \%)\end{array}$ & $\begin{array}{c}123768.3 \\
(9.36 \%)\end{array}$ \\
\hline Etah & 36684.67 & 199869.7 & $\begin{array}{c}22145.33 \\
(-39.63 \%)\end{array}$ & $\begin{array}{c}140363 \\
(-29.77 \%)\end{array}$ \\
\hline Hathras & 8042.667 & 82552 & $\begin{array}{c}25057 \\
(211.55 \%)\end{array}$ & $\begin{array}{l}82031.33 \\
(-0.63 \%)\end{array}$ \\
\hline Shahjehanpur & 164152.67 & 265511.33 & $\begin{array}{c}205205.33 \\
(25.01 \%)\end{array}$ & $\begin{array}{c}250997.33 \\
(-5.47 \%)\end{array}$ \\
\hline Pilibhit & 141211.3 & 137825.3 & $\begin{array}{c}151718.7 \\
(7.44 \%)\end{array}$ & $\begin{array}{r}145779 \\
(5.77 \%)\end{array}$ \\
\hline Badaun & 76496.67 & 266565.3 & $\begin{array}{c}82864.67 \\
(8.32 \%)\end{array}$ & $\begin{array}{l}240563.3 \\
(-9.75 \%)\end{array}$ \\
\hline
\end{tabular}


Table.1 (c) Productivity (Q/ha) of rice and wheat in districts of western UP based on 3-years moving average

\begin{tabular}{|c|c|c|c|c|}
\hline & \multicolumn{2}{|c|}{ 1997-98 } & \multicolumn{2}{|c|}{ 2013-14 } \\
\hline & Rice & Wheat & Rice & Wheat \\
\hline Saharanpur & 25.79 & 28.50 & $\begin{array}{c}25.14 \\
(-2.52 \%)\end{array}$ & $\begin{array}{c}32.53 \\
(14.17 \%)\end{array}$ \\
\hline Baghpat & 23.94 & 35.06 & $\begin{array}{c}27.71 \\
(10.54 \%)\end{array}$ & $\begin{array}{c}40.70 \\
(16.07 \%)\end{array}$ \\
\hline Ghaziabad & 30.57 & 38.38 & $\begin{array}{c}25.93 \\
(6.04 \%)\end{array}$ & $\begin{array}{c}33.00 \\
(-14.02 \%)\end{array}$ \\
\hline Bulandshr & 24.54 & 34.89 & $\begin{array}{c}25.92 \\
(5.60 \%)\end{array}$ & $\begin{array}{c}36.27 \\
(3.96 \%)\end{array}$ \\
\hline Meerut & 24.70 & 36.32 & $\begin{array}{c}28.29 \\
(14.55 \%)\end{array}$ & $\begin{array}{c}38.99 \\
(7.33 \%)\end{array}$ \\
\hline Muzaffarnagar & 27.29 & 31.81 & $\begin{array}{c}24.4 \\
(-10.45 \%)\end{array}$ & $\begin{array}{c}33.3 \\
(4.82 \%)\end{array}$ \\
\hline Bareilly & 22.97 & 25.89 & $\begin{array}{c}21.73 \\
(-5.41 \%)\end{array}$ & $\begin{array}{c}29.72 \\
(14.78 \%)\end{array}$ \\
\hline Rampur & 23.74 & 32.27 & $\begin{array}{c}22.63 \\
(-4.69 \%)\end{array}$ & $\begin{array}{c}33.11 \\
(2.59 \%)\end{array}$ \\
\hline Agra & 20.68 & 34.11 & $\begin{array}{c}25.27 \\
(25.20 \%)\end{array}$ & $\begin{array}{c}32.71 \\
(-4.10 \%)\end{array}$ \\
\hline Mathura & 21.46 & 31.86 & $\begin{array}{c}23.93 \\
(11.51 \%)\end{array}$ & $\begin{array}{c}31.19 \\
(-2.11 \%)\end{array}$ \\
\hline G.B. Nagar & 23.68 & 28.17 & $\begin{array}{c}25.36 \\
(7.08 \%)\end{array}$ & $\begin{array}{c}36.22 \\
(28.56 \%)\end{array}$ \\
\hline Moradabad & 24.11 & 31.27 & $\begin{array}{c}23.01 \\
(-4.56 \%)\end{array}$ & $\begin{array}{c}27.14 \\
(-13.22 \%)\end{array}$ \\
\hline Amroha & 24.66 & 26.10 & $\begin{array}{c}20.77 \\
(-15.76 \%)\end{array}$ & $\begin{array}{c}29.18 \\
(11.80 \%)\end{array}$ \\
\hline Aligarh & 21.99 & 32.25 & $\begin{array}{c}22.51 \\
(2.33 \%)\end{array}$ & $\begin{array}{c}31.30 \\
(-2.94 \%)\end{array}$ \\
\hline Firozabad & 20.92 & 28.98 & $\begin{array}{c}26.83 \\
(28.27 \%)\end{array}$ & $\begin{array}{c}31.25 \\
(7.83 \%) \\
\end{array}$ \\
\hline Mainpuri & 20.96 & 28.12 & $\begin{array}{c}26.23 \\
(25.16 \%)\end{array}$ & $\begin{array}{c}32.39 \\
(15.17 \%)\end{array}$ \\
\hline Bijnor & 29.55 & 26.79 & $\begin{array}{c}25.10 \\
(-15.06 \%)\end{array}$ & $\begin{array}{c}30.66 \\
(14.42 \%)\end{array}$ \\
\hline Etah & 15.21 & 21.87 & $\begin{array}{c}22.94 \\
(50.85 \%)\end{array}$ & $\begin{array}{c}28.93 \\
(32.27 \%)\end{array}$ \\
\hline Hathras & 22.90 & 31.21 & $\begin{array}{c}21.62 \\
(-5.60 \%)\end{array}$ & $\begin{array}{c}32.83 \\
(5.20 \%)\end{array}$ \\
\hline Shahjehanpur & 24.82 & 28.16 & $\begin{array}{c}27.42 \\
(10.48 \%)\end{array}$ & $\begin{array}{c}31.13 \\
(10.55 \%)\end{array}$ \\
\hline Pilibhit & 28.02 & 28.14 & $\begin{array}{c}27.59 \\
(-1.53 \%)\end{array}$ & $\begin{array}{c}36.59 \\
(30.01 \%)\end{array}$ \\
\hline Badaun & 17.83 & 27.84 & $\begin{array}{c}22.46 \\
(25.94 \%)\end{array}$ & $\begin{array}{c}31.16 \\
(11.93 \%)\end{array}$ \\
\hline
\end{tabular}


Table.2 (a) Annual average simple and compound growth rates (\%) of production, area and productivity of Rice

\begin{tabular}{|c|c|c|c|c|}
\hline & & Production & Area & Productivity \\
\hline \multirow[t]{2}{*}{ Saharanpur } & S.G.R. & -1.918 & -1.624 & -0.284 \\
\hline & C.G.R. & -2.105 & -1.475 & -0.652 \\
\hline \multirow[t]{2}{*}{ Baghpat } & S.G.R. & 1.395 & 1.414 & -0.019 \\
\hline & C.G.R. & 2.146 & 1.456 & -0.569 \\
\hline \multirow[t]{2}{*}{ Ghaziabad } & S.G.R. & -0.831 & -1.726 & 0.901 \\
\hline & C.G.R. & -0.756 & -1.564 & 0.845 \\
\hline \multirow[t]{2}{*}{ Bulandshr } & S.G.R. & 9.032 & 8.243 & 0.303 \\
\hline & C.G.R. & 7.125 & 6.147 & 1.023 \\
\hline \multirow[t]{2}{*}{ Meerut } & S.G.R. & -0.038 & -0.79 & 0.785 \\
\hline & C.G.R. & -0.058 & -0.324 & 0.948 \\
\hline \multirow[t]{2}{*}{ Muzafarnagar } & S.G.R. & -5.987 & -5.745 & -0.239 \\
\hline & C.G.R. & -6.229 & -5.622 & -0.135 \\
\hline
\end{tabular}

\begin{tabular}{|l|l|c|c|c|}
\hline \multicolumn{1}{|l|}{ Bareilly } & S.G.R. & Production & Area & Productivity \\
\cline { 2 - 5 } Rampur & C.G.R. & -0.321 & 0.098 & -0.342 \\
\hline \multirow{2}{*}{ Agra } & S.G.R. & -0.194 & 0.141 & -0.238 \\
\hline \multirow{2}{*}{ Mathura } & C.G.R. & -0.126 & 0.407 & -0.529 \\
\hline \multirow{2}{*}{ G.B. Nagar } & S.G.R. & -0.101 & 0.53 & -0.425 \\
\cline { 2 - 5 } & C.G.R. & 14.709 & 13.213 & 1.501 \\
\hline \multirow{2}{*}{ Moradabad } & S.G.R. & 14.936 & 13.336 & 1.605 \\
\hline & C.G.R. & 3.965 & 3.174 & 0.801 \\
\hline & S.G.R. & 4.192 & 3.297 & 0.905 \\
\hline & C.G.R. & 2.913 & 3.349 & -0.436 \\
\hline & S.G.R. & 3.14 & 3.472 & -0.332 \\
\hline & C.G.R. & -1.562 & -0.849 & -0.703 \\
\hline
\end{tabular}

\begin{tabular}{|l|l|c|c|c|}
\hline \multirow{2}{*}{ Amroha } & & Production & Area & Productivity \\
\hline \multirow{2}{*}{ Aligarh } & S.G.R. & -2.11 & -0.997 & -1.118 \\
\cline { 2 - 5 } & C.G.R. & -1.335 & -0.726 & -0.599 \\
\hline \multirow{2}{*}{ Firozabad } & S.G.R. & 8.79 & 8.994 & -0.11 \\
\cline { 2 - 5 } & C.G.R. & 9.017 & 9.117 & -0.006 \\
\hline \multirow{2}{*}{ Mainpuri } & S.G.R. & 5.04 & 1.274 & 4.768 \\
\hline & C.G.R. & 5.267 & 1.397 & 4.872 \\
\hline \multirow{2}{*}{ Bijnor } & S.G.R. & 2.821 & 1.13 & 1.49 \\
\hline \multirow{2}{*}{ Etah } & C.G.R. & 3.048 & 1.253 & 1.594 \\
\hline & S.G.R. & -1.96 & -0.711 & -1.249 \\
\cline { 2 - 5 } & C.G.R. & -1.733 & -0.588 & -1.145 \\
\hline & S.G.R. & -1.415 & -4.229 & 3.079 \\
\hline & C.G.R. & -1.188 & -4.106 & 3.183 \\
\hline
\end{tabular}




\begin{tabular}{|l|l|c|c|c|}
\hline \multirow{2}{*}{ Hathras } & S.G.R. & Production & Area & Productivity \\
\cline { 2 - 5 } & C.G.R. & 7.916 & 7.076 & 0.79 \\
\hline \multirow{3}{*}{ Shahjehanpur } & S.G.R. & 1.143 & 7.199 & 0.894 \\
\cline { 2 - 5 } & C.G.R. & 2.05 & 1.206 & 0.617 \\
\hline \multirow{3}{*}{ Badaun } & S.G.R. & 0.334 & 1.329 & 0.721 \\
\cline { 2 - 5 } & C.G.R. & 0.561 & 0.54 & -0.209 \\
\hline & S.G.R. & 1.065 & -0.483 & -0.105 \\
\cline { 2 - 5 } & C.G.R. & 1.292 & -0.364 & 1.452 \\
\hline
\end{tabular}

Table.2 (b) Annual average simple and compound growth rates (\%) of production, area and productivity of Wheat

\begin{tabular}{|l|l|c|c|c|}
\hline \multirow{4}{*}{ Saharanpur } & S.G.R. & Production & Area & Productivity \\
\cline { 2 - 5 } & C.G.R. & -0.283 & -0.463 & 0.18 \\
\hline \multirow{3}{*}{ Ghazhpat } & S.G.R. & -0.192 & -0.407 & 0.207 \\
\hline \multirow{3}{*}{ Bulandshr } & C.G.R. & 0.961 & 0.022 & 0.939 \\
\hline \multirow{3}{*}{ Meerut } & S.G.R. & 1.052 & 0.078 & 0.966 \\
\cline { 2 - 5 } & C.G.R. & -4.288 & -4.176 & -0.112 \\
\hline \multirow{2}{*}{ Suzafarnagar } & S.G.R. & -4.197 & -4.12 & -0.085 \\
\cline { 2 - 5 } & C.G.R. & 1.14 & 0.906 & 0.234 \\
\hline & S.G.R. & 1.231 & 0.962 & 0.261 \\
\hline & C.G.R. & -0.92 & -0.444 & -0.476 \\
\cline { 2 - 5 } & S.G.R. & -0.829 & -0.388 & -0.449 \\
\cline { 2 - 5 } & C.G.R. & -2.51 & -2.632 & 0.122 \\
\hline
\end{tabular}

\begin{tabular}{|c|c|c|c|c|}
\hline & & Production & Area & Productivity \\
\hline \multirow[t]{2}{*}{ Bareilly } & S.G.R. & 1.232 & 0.863 & 0.369 \\
\hline & C.G.R. & 1.323 & 0.919 & 0.396 \\
\hline \multirow[t]{2}{*}{ Rampur } & S.G.R. & 0.618 & 1.263 & -0.645 \\
\hline & C.G.R. & 0.709 & 1.319 & -0.618 \\
\hline \multirow[t]{2}{*}{ Agra } & S.G.R. & 0.509 & 0.536 & -0.027 \\
\hline & C.G.R. & 0.6 & 0.592 & -0.015 \\
\hline \multirow[t]{2}{*}{ Mathura } & S.G.R. & 0.618 & 0.079 & 0.539 \\
\hline & C.G.R. & 0.709 & 0.135 & 0.551 \\
\hline \multirow[t]{2}{*}{ G.B. Nagar } & S.G.R. & -2.249 & -3.125 & 0.876 \\
\hline & C.G.R. & -2.158 & -3.069 & 0.888 \\
\hline \multirow[t]{2}{*}{ Moradabad } & S.G.R. & -2.602 & -1.87 & -0.732 \\
\hline & C.G.R. & -2.511 & -1.881 & -0.720 \\
\hline
\end{tabular}




\begin{tabular}{|l|l|c|c|c|}
\hline \multirow{2}{*}{ Amroha } & S.G.R. & Production & Area & Productivity \\
\cline { 2 - 5 } & C.G.R. & 0.261 & -0.178 & 0.439 \\
\hline \multirow{2}{*}{ Figarozabad } & S.G.R. & 0.84 & -0.189 & 0.451 \\
\cline { 2 - 5 } & C.G.R. & 0.931 & 0.816 & 0.024 \\
\hline \multirow{2}{*}{ Mainpuri } & S.G.R. & 0.912 & 0.805 & 0.036 \\
\cline { 2 - 5 } & C.G.R. & 1.003 & 0.191 & 0.71 \\
\hline \multirow{2}{*}{ Bijnor } & S.G.R. & 2.101 & 1.33 & 0.722 \\
\cline { 2 - 5 } & C.G.R. & 2.192 & 1.319 & 0.771 \\
\hline \multirow{2}{*}{ Etah } & S.G.R. & 1.137 & 0.255 & 0.783 \\
& C.G.R. & 1.228 & 0.244 & 0.882 \\
\hline & S.G.R. & -1.625 & -2.766 & 1.141 \\
\cline { 2 - 5 } & C.G.R. & -1.534 & -2.777 & 1.153 \\
\hline
\end{tabular}

\begin{tabular}{|l|l|c|c|c|}
\hline \multirow{2}{*}{ Hathras } & & Production & Area & Productivity \\
\hline \multirow{2}{*}{ Shahjehanpur } & C.G.R. & -0.526 & -0.339 & -0.187 \\
\cline { 2 - 5 } & S.G.R. & -0.435 & -0.35 & -0.175 \\
\cline { 2 - 5 } Pilibhit & C.G.R. & 0.749 & -0.159 & 0.908 \\
\hline \multirow{2}{*}{ Badaun } & S.G.R. & 0.84 & -0.17 & 0.920 \\
\cline { 2 - 5 } & C.G.R. & 1.469 & 0.406 & 1.063 \\
\hline & S.G.R. & 1.56 & 0.395 & 1.075 \\
\cline { 2 - 5 } & C.G.R. & 0.453 & -0.548 & 1.001 \\
\hline
\end{tabular}

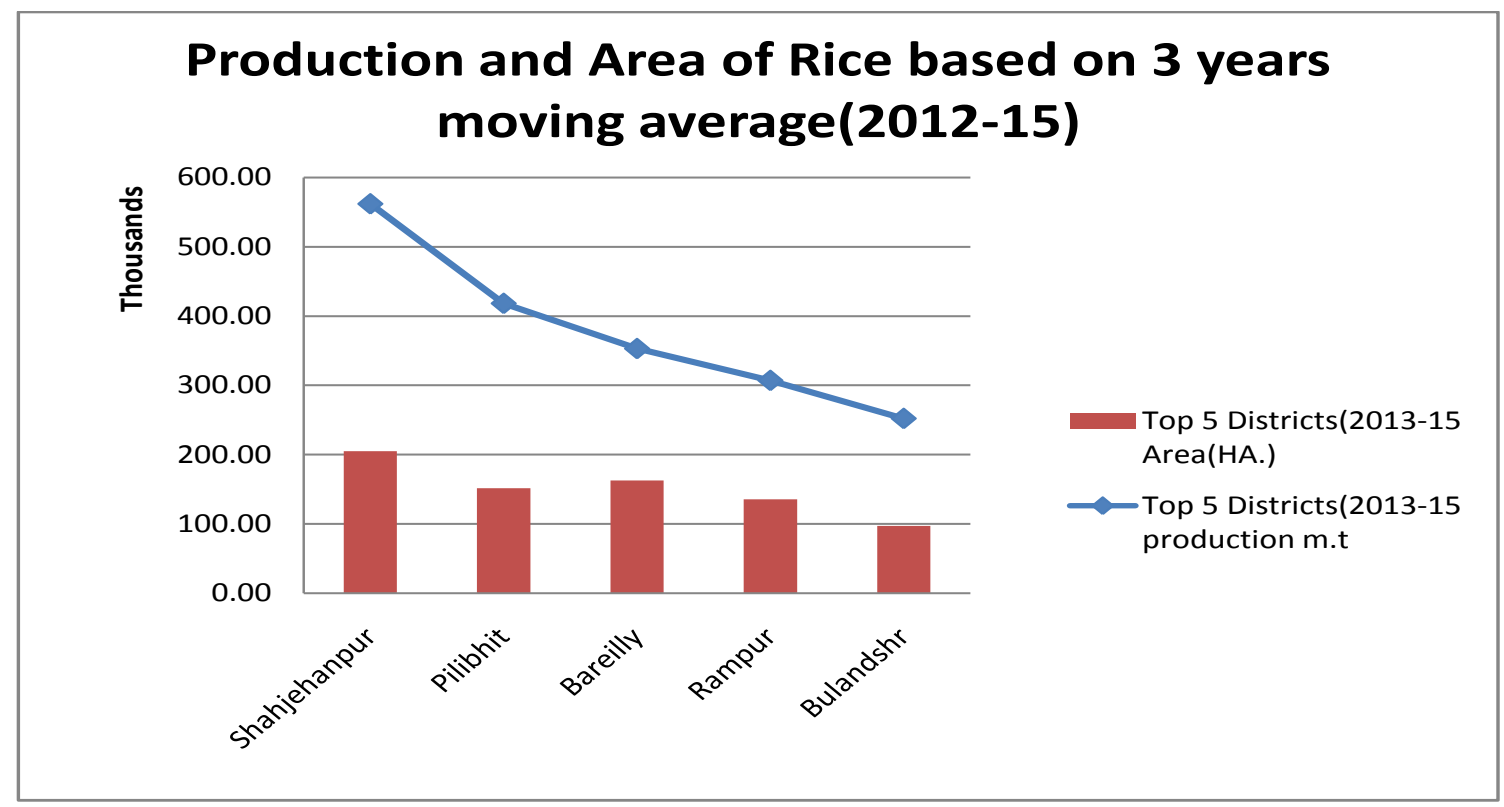

The favourable conditions like Climate, Rainfall and Temperature along with Tarai Soils attributes to more Production and Area in Shahjehanpur, Pilibhit \& Bareilly Districts than other Districts of western U.P. 


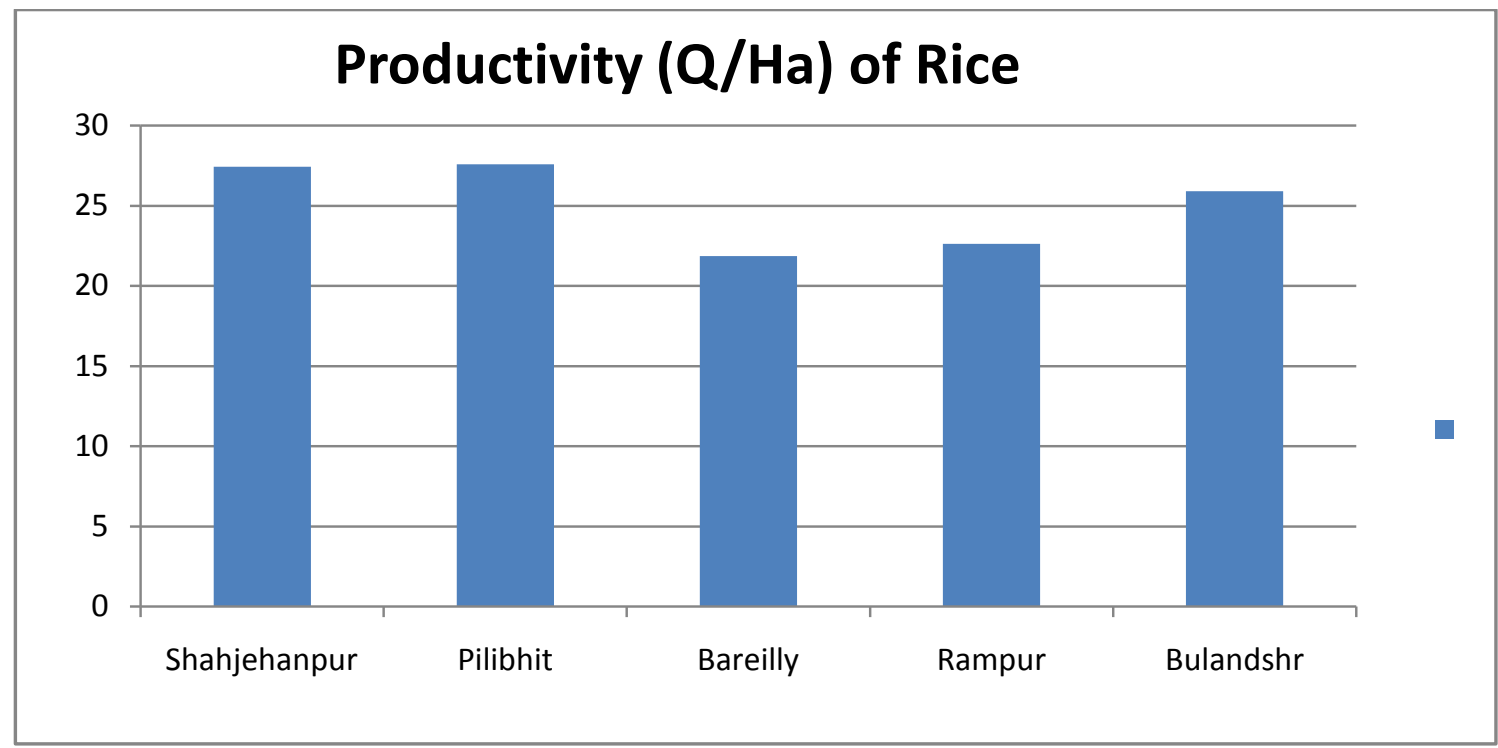

The Productivity of Rice is highest in Shahjehanpur,Pilibhiit and Barelliy is due to present of Watterlogged Tarai Soil , favourable climatic conditions and more fertilizer use in these districts. Although the Area \& Production of Rice in Shajehanpur is much more than Pilibhit district, the productivity in Pilibhit is somewhat larger than in Shahjehanpur.

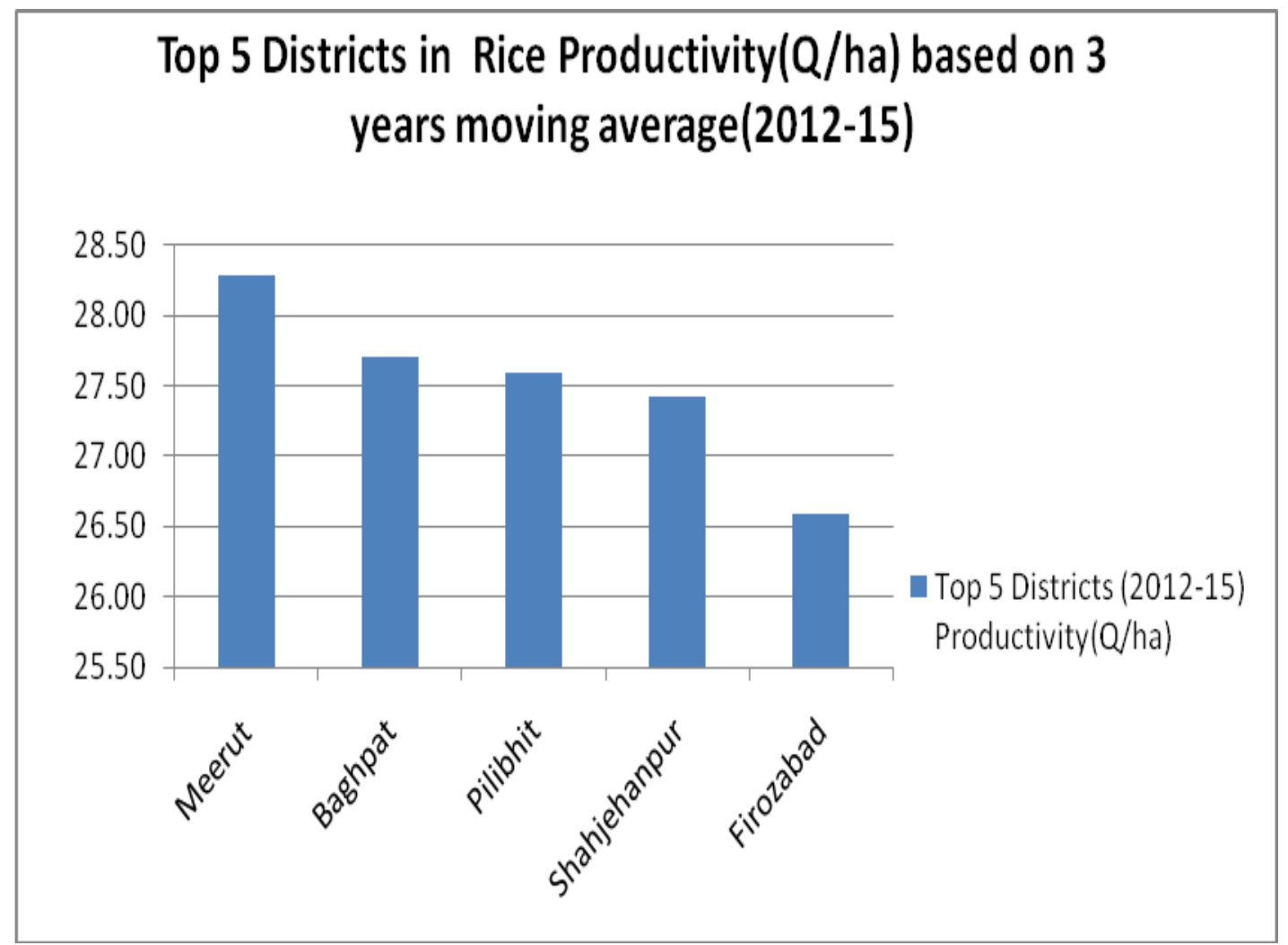




\section{Production and Area of wheat based on 3 years moving average(2012-15)}

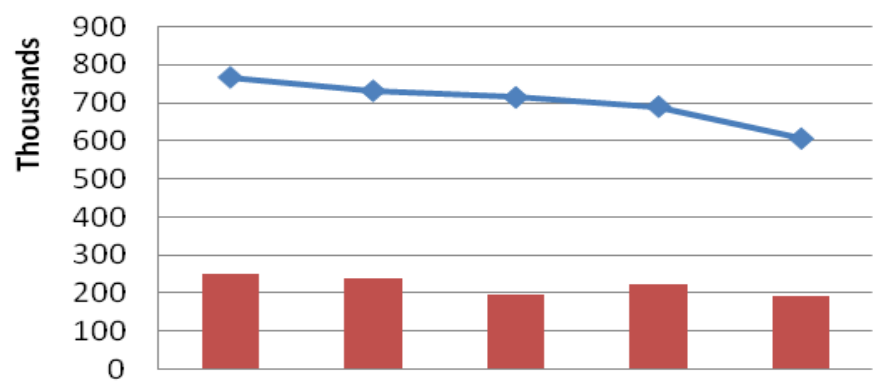

Top 5 Districts(2013-15 Area(HA.)

—Top 5 Districts(2013-15 production m.t

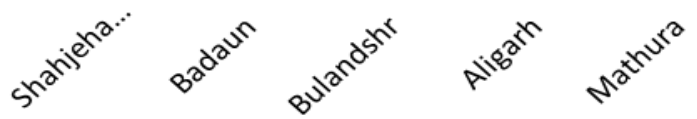

\section{Productivity(Q/ha) of Wheat}
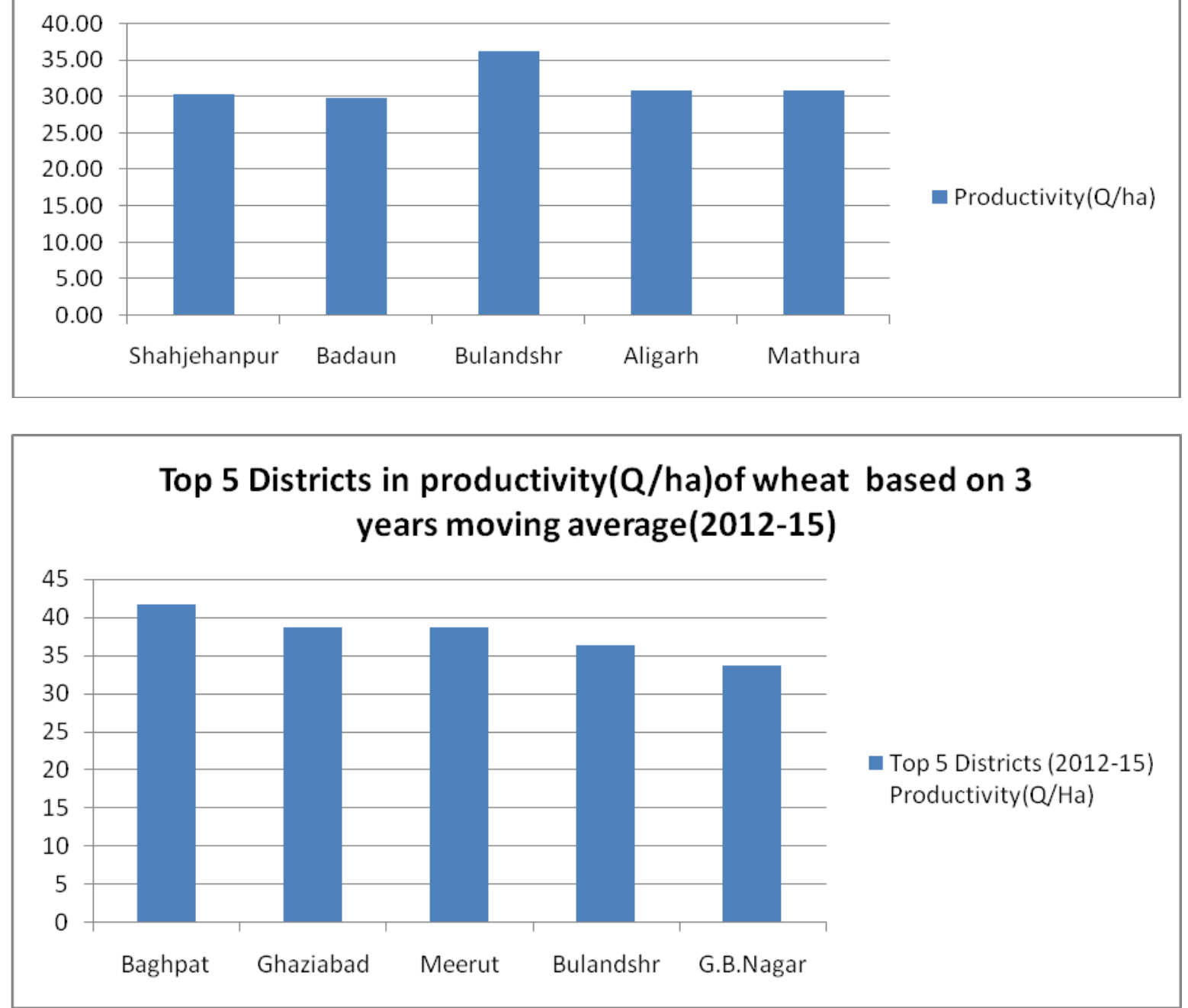
Highest percent change shown by Etah district in Rice followed by Firozabad and Agra district.

Highest percent change shown by Etah district in wheat followed by Pilibhit and G.B. Nagar district.

Productivity of Rice declined being maximum in Amroha followed by Bijnor and Muzaffarnagar.

Productivity of Wheat declined being maximum in Ghaziabad followed by Moradabad and Agra.

Apart from this the Productivity of Rice is maximum in Meerut and Baghpat Districts indicating the presence of more waterlogged soil and soil fertility. As compared to Punjab (33.37 Q/ha) Rice productivity in western U.P. has far less productivity (20.42 Q/ha). This shows that rice is more intensively cultivated in Punjab. The graph shows that the area and production under wheat is highest in Shahjehanpur and Badaun Districts but its productivity is highest in Bulandshr district.

Among all states of western U.P., the Baghpat records the highest wheat productivity. As compared to Punjab the average productivity of western U.P. (28.61 Q/ha) is far less than (44.9 Q/ha) of Punjab indicating the more sources of irrigation and fertilizer-use than Western U.P.

\section{The second objective}

To obtain growth rates of production, area and productivity of rice and wheat crops in districts of western U.P. based on timeseries data

Annual growth rates of area, Production and productivity of Rice and Wheat crops have worked out using two functions, viz. Simple and compound growth for the districts of western U.P. The results are presented and discussed in this section.

Perusal of above Table 2(a), The present investigation showed that in rice crop the highest SGR and CGR in case of Production 14.709 and 14.936 percent respectively in Agra district followed by Bulandshr and Aligarh district whereas, for the area found maximum in Agra i.e. 13.213 and 13.336 percent respectively followed by Aligarh and Bulandshr district. In case of Productivity it is Firozabad followed by Etah and Agra district.

Perusal of above Table 2(b), The present investigation showed that in wheat crop the highest SGR and CGR in case of Production 2.101 and 2.192 percent respectively in Mainpuri district followed by Pilibhit and Bareilly district whereas, for the area found maximum in Mainpuri i.e. 1.33 and 1.319 percent respectively followed by Pilibhit and Rampur district. In case of Productivity it is G.B. Nagar followed by Pilibhit and Badaun district.

\section{References}

Neeraj Singh, Piyush Kumar Singh and Sunil Kumar (2018): Growth Rate of Wheat Crop in Azamgarh Division of Eastern Uttar Pradesh, India. Int.J.Curr.Microbiol.App.Sci. 7(03):3348-3352

Piyush Kumar Singh, Neeraj Singh, Sunil Kumar and V.D. Chaturvedi (2018): Growth rate of Wheat crop in Varanasi division of Eastern Uttar Pradesh, India Int.J.Curr.Microbiol.App.Sci.

7(05):1939-1944

Prajneshu and K. P. Chandran (2005) Computation of Compound Growth Rates in Agriculture. Agricultural Economics Research Review, Vol. 18: 317-324. 
Singh, R. P. (2003) Analysis of Growth Performance of Wheat Crop in Jharkhand; J. Res. Birsa Agri. Univ., 15(2): 217-223.

Srivastava S.C., Singh, B.K., Sudeep S Tomar and Yadava, H.S (2013) Growth Pattern of Major Pulses in Eastern Uttar Pradesh. International Journal of Farm Sciences, 3(2): 1-9.

\section{How to cite this article:}

Gopal Krishna Tiwari, Sri Sunil Kumar, Anvesha Singh and Babulal. 2019. A Statistical Study of Trends of Rice-Wheat Production on Districts of Western U.P., India Int.J.Curr.Microbiol.App.Sci. 8(06): 2430-2443. doi: https://doi.org/10.20546/ijcmas.2019.806.290 